\title{
Effects of a Novel Whitening Formulation on Dental Enamel
}

\section{Thair Takesh, Anik Sargsyan, Afarin Anbarani, Jessica Ho and Petra Wilder-Smith}

Beckman Laser Institute, University of California, Irvine CA 92617

*Corresponding author: Petra Wilder-Smith, Professor, Director of Dentistry; Beckman Laser Institute, University of California, Irvine; 1002 Health Sciences Rd East, Irvine, CA 92617, USA, Tel: +1 949-824-5011; E-mail: pwsmith@uci.edu

Received date: March 06, 2017; Accepted date: March 27, 2017; Published date: April 03, 2017

Copyright: (C) 2017 Takesh T, et al. This is an open-access article distributed under the terms of the Creative Commons Attribution License, which permits unrestricted use, distribution, and reproduction in any medium, provided the original author and source are credited.

\begin{abstract}
Objective: The goal of this study was to evaluate the enamel whitening effects of 2 new test formulations, one of which was a rinse, and the other a whitening strip.

Materials and Methods: Forty enamel chips were prepared from 20 healthy extracted teeth (2 from each tooth). After pre-staining and colorimetry to measure $\mathrm{L}^{*}$ and $\mathrm{b}^{*}$ values, 20 matched samples were immersed in either test or control rinses, and then colorimetry was performed again after $1 \mathrm{hr}, 2 \mathrm{hr}, 3 \mathrm{hr}, 6 \mathrm{hr}, 12 \mathrm{hr}, 24 \mathrm{hr}$ and $48 \mathrm{hrs}$ (Each hour equates to one month of clinical use at the recommended dosage of 1 minute exposure 2 times a day). The remaining 20 matched samples were exposed to the test or control whitening strips and colorimetry was performed every 30 minutes for a total of 10 treatments.
\end{abstract}

Results: Overall, the whitening performance of test and control strips was similar. The test and control rinses had a similar lightening effect over the first 3 hours (equivalent to 3 months of clinical use). Subsequently, the control rinse continued to lighten samples, whereas the test rinse had little further effect.

Conclusion: Test and control-whitening strips showed similar effects; over time whitening strips showed a greater lightening effect than whitening rinses.

Keywords: Whitening; Enamel; Bleaching; Mouth rinse; Bleaching strips; Colorimetry

\section{Introduction}

A recent survey showed $80 \%$ of Americans aged 18 to 49 want whiter teeth1. According to the American Academy of Cosmetic Dentistry, Americans spent more than $\$ 1.4$ billion on over-the-counter teeth whitening products last year alone. Tooth bleaching has become the most popular cosmetic procedure offered in dental practice [1]. Extrinsic stains usually result from the accumulation on the external tooth surface of chromatogenic substances from sources such as poor oral hygiene, ingestion of chromatogenic food and drinks, and tobacco use. These stains are localized mainly on the surface, and are either generated by the reaction between sugars and amino acids or acquired from the retention of exogenous chromophores in the pellicle [2]. "Over-the-counter" (OTC) tooth bleaching agents were first launched in the United States in the 1990s. They contain lower concentrations of hydrogen peroxide or carbamide peroxide than professional whitening formulations [3].

Current in-home bleaching agents contain both active and inactive substances. The active ingredients are usually hydrogen peroxide or carbamide peroxide compounds. Inactive components may include thickening agents, carrier, surfactant and pigment dispersant, preservative, and flavoring [4]. Side-effects of in-home bleaching may include soft-tissue irritation, tooth sensitivity and dental erosion [5]. These changes can be greater when the contact time between the bleaching agent and the tooth surface is increased [6]. However, studies have shown that the addition of calcium or fluoride to the composition of a bleaching agent can minimize mineral loss in the enamel $[7,8]$.

Goal of this ex vivo study was to identify the whitening effects on tea-stained dental enamel of 2 new test formulations, one of which was a rinse, and the other a whitening strip. The test formulations contain natural ingredients only, and are free of peroxide compounds [9-11].

\section{Materials and Methods}

\section{Samples}

A total of 40 enamel samples approx. $5 \mathrm{~mm} \times 5 \mathrm{~mm} \times 3 \mathrm{~mm}$ in size were prepared from sterilized extracted teeth that had been classified as healthy by an experienced dentist using a loupe and headlamp. Two chips were obtained from each tooth. To prevent exposure of the cut surfaces of the samples to the staining and bleaching agents, the samples were affixed to a strip of impermeable plastic. All samples were stained by overnight immersion in a concentrated black tea solution produced by steeping 2 teabags (Lipton'sR yellow label black tea) in $1 / 2$ cup of boiling water for 30 minutes. Each sample pair was stained in a separate vial. Then paired samples were randomly divided into 2 groups of 10 pairs each. The $\mathrm{L}^{*}$ and $\mathrm{b}^{*}$ color values of each sample were recorded under carefully standardized lighting conditions using a reflectance spectrophotometer Colorimeter (PCE-CSM 1, PCE Instruments ${ }^{\mathrm{m}}$, PCE Instrumentation, Alicante, Spain) and then photographed individually under the same lighting conditions at each study time point.

Group 1: One stained chip (total 10 samples) from each pair was treated with test whitening strips (Oral EssentialsR Whitening strips, 
Oral Essentials, Beverly Hills, CA); the matching second chip was exposed to the control strip (3D Crest White stripsR, Procter \& Gamble Company, Cincinnati, OH). Data were collected immediately after staining, and again after 60 mins of whitening strip application. This was repeated 10 times. Samples underwent a 1 minute immersion in saliva solution after each bleaching episode to eliminate any temporary enamel white spots that might affect colorimetry results.

Group 2: One stained chip (total 10 samples) from each pair was immersed in the test whitening rinse (Oral EssentialsR whitening formula mouthwash, Oral Essentials, Beverly Hills, CA) and the matching second chip was exposed to the control whitening rinse (Crest 3D whitening mouthwashR, Procter \& Gamble Company, Cincinnati, OH). Sample color was recorded by colorimetry and photographs at the following time points: baseline, after $1 \mathrm{hr}, 2 \mathrm{hr}, 3 \mathrm{hr}$, $6 \mathrm{hr}, 12 \mathrm{hr}, 24 \mathrm{hr}$ and $48 \mathrm{hr}$ immersion in whitening rinse. Each hour of immersion equates to 1 month of clinical use at the recommended dosage of 1 minute exposure 2 times a day (personal communication, Dr Kourosh Maddahi, Oral Essentials, Beverly Hills, CA).

\section{Colorimetry}

A reflectance spectrophotometer was used to measure enamel color. It has a $4 \mathrm{~mm}$ diameter measuring area and a D-65 standard illuminant. The image of the tooth specimen is automatically transformed to derive a set of numerical color values in terms of the $L^{*} b^{*}$ system. The $L^{*}$ value represents a sample's degree of lightness, ranging from 0 (black) to 100 (white). The $b^{*}$ value quantifies the blue/ yellow color component of the sample, whereby a value of 0 indicates an equal presence of both colors; negative scores represent a predominantly blue component value, whereas positive scores indicate a stronger yellow color component. When evaluating tooth whitening, a yellow hue (positive $b^{*}$ score) is generally considered more desirable than a blue one (negative $b^{*}$ score).

\section{Statistical evaluation}

A two-tailed T-test was used to compare results for control vs test agent within each agent group (strips and mouth rinse).

\section{Results}

\section{Group 1: whitening strips}

The test strip formulation whitened only the enamel surface of the samples, and had no visible effect on the underlying dentin. Figure 1 shows a comparison of the effects of each whitening strip treatment on $L^{*}$ value and $b^{*}$ values respectively.
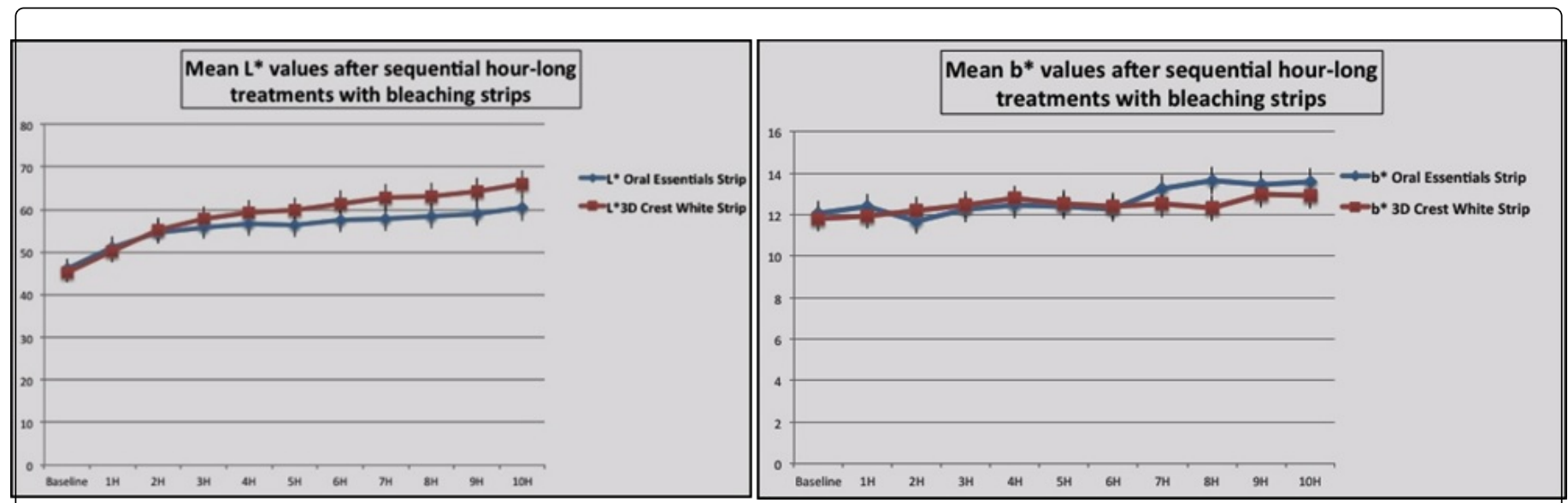

Figure 1: Effects of each whitening strip treatment on $\mathrm{L}^{*}$ value (LHS) and $b^{*}$ values (RHS) respectively.

$\mathrm{L}^{*}$ value measures: Baseline $\mathrm{L}^{*}$ values for the 2 groups did not differ significantly $(p>0.05)$. The effects of the 2 strip formulations were statistically identical over the first 4 hours; then the test strips lagged by less than $15 \%$ at each measurement time point. Given a mean standard deviation of $9 \%$, these differences are barely significant $(\mathrm{p}<0.05)$.

$b^{*}$ value measures: Baseline $b^{*}$ values for the 2 groups did not differ significantly $(p>0.05)$. The effects of the 2 strip formulations were statistically identical over the entire test period $(\mathrm{p}>0.05)$ except at $8 \mathrm{~h}$, when the test formulation was marginally better than the control. Most likely this is an inconsequential minor outlier of the data, as the difference barely exceeds the mean standard deviation. For all samples, the $b^{*}$ values increased to a small extent over time, indicating a mild shift towards the yellow and away from the blue.

\section{Group 2: whitening rinse}

The mouth rinses whitened the surface enamel and the underlying dentin also. Figure 2 shows a comparison of the effects of each whitening rinse treatment on $\mathrm{L}^{*}$ value and $\mathrm{b}^{*}$ values respectively. 


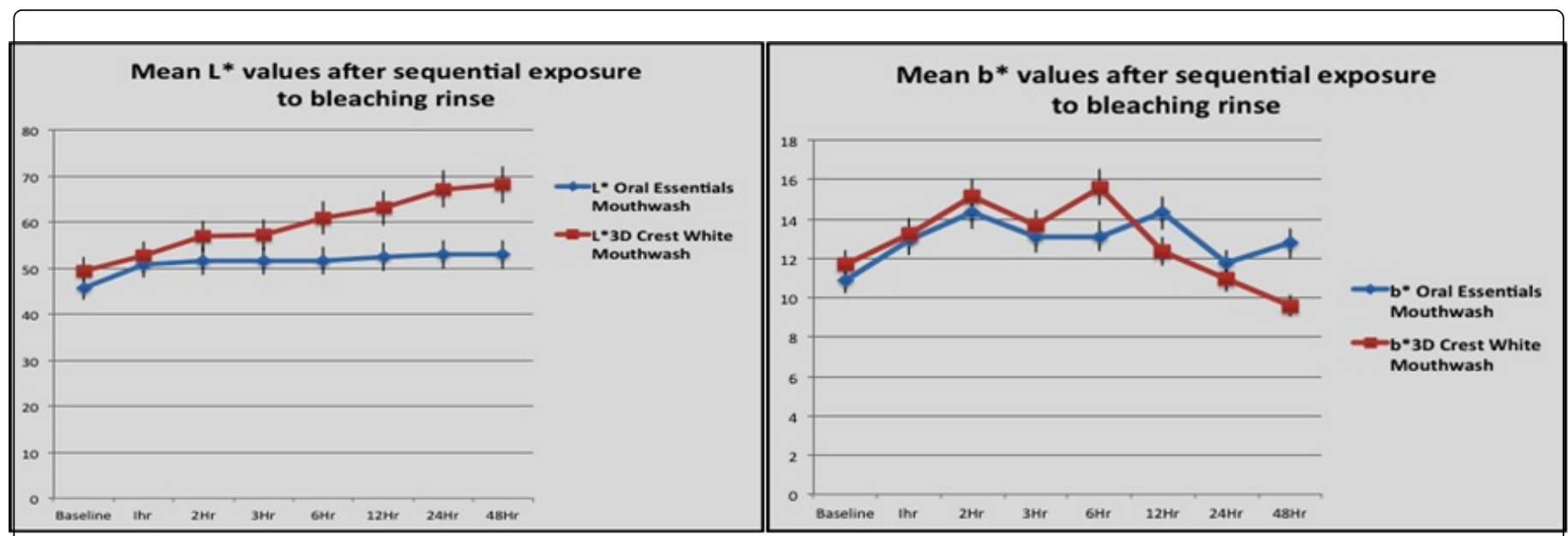

Figure 2: Effects of each whitening rinse treatment on $\mathrm{L}^{*}$ value (LHS) and $\mathrm{b}^{*}$ values (RHS) respectively.

$\mathrm{L}^{*}$ value measures: Baseline $\mathrm{L}^{*}$ values for the 2 groups did not differ significantly $(p>0.05)$. The test formulation achieved close to maximum lightening effect after 1 hour of exposure, with additional bleaching duration adding approximately $6 \%$ of additional lightening effect. This additional increase lies within the SD range, and is statistically not significant $(\mathrm{p}>0.05)$. The bleaching performance of the 2 solutions did not differ significantly over the first 3 hours $(p>0.05)$. After this time point, the test solution lagged significantly, with the control solution providing more than twice as much lightening effect as the test agent after 24 hours $(\mathrm{p}<0.05)$.

$b^{*}$ value measures: Baseline $b^{*}$ values for the 2 groups did not differ significantly $(p>0.05)$. The effects of the 2 formulations were statistically identical over the entire test period ( $>>0.05)$, except at $6 \mathrm{~h}$ and $48 \mathrm{~h}$. At $6 \mathrm{~h}$ the samples exposed to the control solution showed a somewhat greater shift to a yellow hue compared to those exposed to the test solution, whereas at $48 \mathrm{~h}$ the samples treated with the test solution were somewhat more yellow.

\section{Discussion}

Overall, the whitening strips showed a more consistent lightening effect than the whitening rinses. The test rinse achieved a more attractive end shade for the tooth surface than the control rinse, in that samples had less of a blue-tinge. This is clinically desirable, as a blue hue adds a harsh, unnatural appearance to the teeth. Perhaps the observed difference in final enamel hues between rinses and strips can in part be attributed to the fact that the rinses bleached both the enamel and the dentin samples, giving them more of a blue-ish appearance, while the strips bleached enamel without much effect on the dentin, allowing the dentin color to shine through the enamel and soften its color. As dentin is more yellow in color than enamel, any dentin bleaching effect would shift overall sample hue away from yellow and more into the blue range.

A previous clinical study comparing the whitening efficacy of a mouth rinse vs. a strip also found greater improvement in $\mathrm{L}^{*}$ value after 8 days of use of a $10 \%$ hydrogen peroxide whitening strip compared to $2 \%$ hydrogen peroxide mouth rinse 9 . The mouth rinse group had a significant increase in yellow vs. blue color balance by day 8 , confirming the results of our study that the rinse produces a more pleasing, less blue tint than the whitening strips. Another study10 compared the whitening effects of $6 \%$ carbamide peroxide strips vs. those of a combination $10 \%$ carbamide peroxide/dentifrice/mouth rinse tooth-whitening system. The strips produced an almost three times greater reduction in the $b^{*}$ yellow hue and almost doubled $L^{*}$ lightness values compared to the tray-based combination system. Again, as in our research, use of the strips in this study resulted in a more blue-toned, lighter final hue of the teeth than the mouth rinse lightening system.

In a metanalysis of eight studies 11 comparing the efficacy and safety of over-the-counter home whitening strips vs. $10 \%$ carbamide peroxide gel, no significant difference for tooth-whitening efficacy measured as $\mathrm{L}^{*}$ was determined between the intervention groups. Again the reported increase in blue tone $\mathrm{b}^{*}$ was more marked after the use of whitening strips, supporting the findings of our study.

\section{Conclusion}

A novel whitening strip showed a comparable lightening effect to a popular OTC whitening strip. Generally, the strips had a greater whitening effect than the rinses. The test rinse achieved a pleasing end color with less blue-tinge than the control rinse. Further studies are required to determine the effects of this novel formulation on tooth sensitivity, gingival health and the enamel surface.

\section{Acknowledgements}

This research was supported by funding from: LAMMP NIH/NIBIB P41EB05890; NIH/NIBIB R03EB014852; the Arnold and Mabel Beckman Foundation; University of California SOM Seed Grant; Oral Essentials Inc.

\section{References}

1. Kihn PW (2007) Vital tooth whitening. Dent Clinic North Am 51: 319-331.

2. Viscio D, Gaffar A, Fakhry-Smith S, Xu T (2000) Present and future technologies of tooth whitening. Compend Contin Educ Dent Suppl 28:S36-S43.

3. Greenwall L, Fredman G, Gordan VV, Martin K, Gerald M, et al. (2001) Bleaching Techniques in Restorative Dentistry: An Illustrated Guide. Martin Dunitz Ltd. 
Citation: Thair Takesh, Anik Sargsyan, Afarin Anbarani, Jessica Ho, Petra Wilder-Smith (2017) Effects of a Novel Whitening Formulation on Dental Enamel . Dentistry 7: 424. doi:10.4172/2161-1122.1000424

Page 4 of 4

4. lqahtani M Q (2014) Tooth-bleaching procedures and their controversial effects: A literature review. Saudi Dent J 26: 33-46.

5. Li Y (1997) Toxicological considerations of tooth bleaching using peroxide-containing agents. J Am Dent Assoc 128: 31S-36S.

6. Mondelli RF, Azevedo JF, Francisconi PA, Ishikiriama SK, Mondelli J (2009) Wear and Surface roughness of bovine enamel submitted to bleaching. Eur J Esthet Dent 4: 396-403.

7. Cavalli V, Rodrigues LK, Paes-Leme AF (2010) Effects of bleaching agents containing fluoride and calcium on human enamel. Quintessence Int 41: e157-165.

8. Chen HP, Chang CH, Liu JK, Chuang SF, Yang JY (2008) Effect of fluoride containing bleaching agents on enamel surface properties. J Dent 36: 718-725.
9. Gerlach RW, Tucker HL, Anastasia MK, Barker ML (2005) Clinical trial comparing 2 hydrogen peroxide tooth whitening systems: strips vs prerinse. Compend Contin Educ Dent. 26: 874-878.

10. Karpinia K, Magnusson 1, Barker ML, Gerlach RW (2003) Clinical comparison of two self-directed bleaching systems. Journal of Prosthodontics 12: 242-248.

11. Serraglio CR, Zanella L, Battestin K, Dalla-Vecchia KB, Rodrigues SA (2016) Efficacy and safety of over-the-counter whitening strips as compared to home-whitening with $10 \%$ carbamide peroxide gelsystematic review of RCTs and meta analysis. Clinical Oral Investigation 20: $1-14$. 